\title{
An Example on Sobolev Space Approximation
}

\author{
Anthony G. O'Farrell
}

St. Patrick's College, Maynooth, Co. Kildare, Ireland

Abstract. For each $d \geq 2$ we construct a connected open set $\Omega \subset{ }^{d}$ such that $\Omega=\operatorname{int}(\operatorname{clos}(\Omega))$ and for each $k \geq 1$ and each $p \in[1,+\infty)$, the subset $W^{k, \infty}(\Omega)$ fails to be dense in the Sobolev space $W^{k, p}(\Omega)$, in the norm of $W^{k, p}(\Omega)$.

1991 MSC Classification: 46J 


\section{Introduction.}

We use to denote the set of real numbers, for the natural numbers, and by a domain we mean a connected open set.

For each open set $\Omega \subset^{d}$, and for each $k \in$ and $p \geq 1$, let $W^{k, p}(\Omega)$ denote the (Sobolev) space of all those distributions $f \in C_{\mathrm{cs}}^{\infty}(\Omega)^{\prime}$ such that $f$ and all its distributional partial derivatives $\partial^{i} f$ of order $|i| \leq k$ are representable by integration against functions belonging to $L^{p}(\Omega, d x)$. When endowed with the norm

$$
f \mapsto\|f\|_{W^{k, p}(\Omega)}=\sum_{0 \leq|i| \leq k}\left\|\partial^{i} f\right\|_{L^{p}(\Omega)},
$$

the space $W^{k, p}(\Omega)$ becomes a Banach space (cf. [1]). These spaces are useful in the theory of partial differential equations, and have been much studied.

In general, $C^{\infty}(\Omega) \cap W^{k, p}(\Omega)$ is dense in $W^{k, p}(\Omega)$ when $p<+\infty$ (and is weak-star dense when $p=+\infty)$, but it may happen that $C_{\mathrm{cs}}^{\infty}(\Omega)$ is not dense in $W^{k, p}(\Omega)$. It may even happen that $C_{\mathrm{cs}}^{\infty}\left({ }^{d}\right) \mid \Omega$ is not dense in $W^{k, p}(\Omega)$, even when $\Omega$ is a bounded domain. A simple example, as Adams first observed (cf. [6]), is the slit disk. It is less easy to see what happens when $\Omega=\operatorname{clos}(\operatorname{int}(\Omega))$. Fraenkel posed the question in his 1979 'rooms and passages' paper [3], and Amick [2] provided an example in 2 dimensions that works whenever $k p>2$. In 1981, Kolsrud showed how to make an example for any $p \geq 1$ and $k \geq 1$. In both cases, the examples given were such that even $C(\operatorname{clos} \Omega) \cap W^{k, p}(\Omega)$ fails to be dense in $W^{k, p}(\Omega)$.

There were also some positive results. For bounded domains with very nice boundary it is true that all functions belonging to $W^{k, p}(\Omega)$ have extensions in $W^{k, p}\left({ }^{d}\right)$, and hence may be approximated (in norm for $p<+\infty$, and weak-star for $p=+\infty$ ) by 
smooth functions; one uses mollifiers. In 1981, Jones [4] established the $W^{k, p}$ extension theorem for the so-called $(\epsilon-\delta)$ domains, which include some rather badly-bounded domains such as general quasi-disks in ${ }^{2}$, so this entails that $C^{\infty}\left({ }^{d}\right) \mid \Omega$ is dense in $W^{k, p}(\Omega)$ for $(\epsilon-\delta)$ domains. Subsequently, in 1987, Lewis [7] showed that $C^{\infty}\left({ }^{d}\right) \mid \Omega$ is dense in $W^{1, p}(\Omega)$ whenever $\Omega$ is a Jordan domain in ${ }^{2}$, and he asked [6] whether this holds for general $W^{k, p}$.

Given that $C(\operatorname{clos} \Omega) \cap W^{k, p}(\Omega)$ need not always be dense in $W^{k, p}(\Omega)$, it became interesting to ask whether $W^{k, \infty}(\Omega)$ is always dense in $W^{k, p}(\Omega)$ when $p<+\infty$. I understand that this question was first raised by Vodopyanov. The examples of Amick and Kolsrud do not settle this question, because in them $W^{k, \infty}(\Omega)$ is not contained in $C(\operatorname{clos} \Omega) \mid \Omega$. In 1994, Tartar (private communication) constructed na example for cases of $(d, k, p)$ such that $W^{k, p}\left({ }^{d}\right) \subset C\left({ }^{d}\right)$, and Vodopyanov (private communication), independently, found an example subject to the same restriction. It was suspected that this restriction was essential, and that for $p<d / k$ one would find that for each bounded domain $\Omega$, with $\Omega=$ int $\operatorname{clos} \Omega$, the space $W^{k, \infty}(\Omega)$ would be dense in $W^{k, p}(\Omega)$.

The purpose of this paper is to show that this suspicion is unfounded: for each $d \geq 2$, there exists a bounded domain $\Omega$, with $\Omega=\operatorname{int} \cos \Omega$, such that for each $p \in[1,+\infty)$ and each $k \geq 1$, the space $W^{k, \infty}(\Omega)$ fails to be dense in $W^{k, p}(\Omega)$; in fact, we shall produce an $\Omega$ and a function

$$
f \in \bigcap_{\substack{k \geq 1 \\ 1 \leq p<\infty}} W^{k, p}(\Omega)
$$

such that no sequence $g_{n} \in W^{1, \infty}(\Omega)$ converges to $f$ in $W^{1,1}(\Omega)$ norm. 


\section{The Construction.}

The ingredients of the construction are Fraenkel's 'rooms and passages' idea [3] and an idea like Kolsrud's use of the well-known 'string of beads'. In fact, it is more convenient to use a 'string of diamonds' (or, when $d \geq 3$, a 'field of cones'), because it has a Lipschitz upper and lower boundary, and this allows us to establish the properties of the example very quickly by appealing to the substantial body of results now known about domains with Lipschitz boundaries.

Let ${ }_{d}(a, r)$ denote the closed ball in ${ }^{d}$ having centre $a$ and radius $r$, and let ${ }_{d}(a, r)$ denote the corresponding open ball. Regard $T={ }_{d-1}(0,1)$ as the closed equatorial plane of ${ }_{d}(0,1)$, i.e.

$$
T=\left\{\left(x_{1}, \ldots, x_{d}\right) \in{ }_{d}(0,1): x_{d}=0\right\}
$$

Choos pairwise-disjoint closed balls

$$
B_{n}={ }_{d-1}\left(a_{n}, r_{n}\right) \subset_{d-1}(0,1),
$$

such that $\bigcup_{n=1}^{\infty} B_{n}$ is dense in $T$ and

$$
\sum_{n=1}^{\infty} r_{n}^{d-1}<1 .
$$

This guarantees that the set

$$
E=T \sim \bigcup_{n=1}^{\infty} B_{n}
$$

has positive $(d-1)$-dimensional volume, $\mathcal{H}^{d-1}(E)$. We further assume that $r_{n}<1 / 2$ for each $n$.

On each $(d-1)$-dimensional $B_{n}$, erect the diamond, or cone:

$$
D_{n}=\left\{(x, z) \in^{d-1} \times: x \in B_{n},|z| \leq \operatorname{dist}\left(x, S_{n}\right)\right\},
$$


where $S_{n}$ denotes the boundary of $B_{n}$, a sphere of dimension $d-2$.

Let

$$
H=T \cup \bigcup_{n=1}^{\infty} D_{n} .
$$

Then $H$ is a compact subset of $B_{d}(0,1)$, and $E \subset H$. We note further that

$$
H \subset{ }_{d}(0,1) \cup^{d-2},
$$

where ${ }^{d-2}=\left\{(x, 0) \in^{d-1} \times:|x|=1\right\}$.

Let $\Omega_{0}={ }_{d}(0,1) \sim H$. Then $\Omega_{0}$ is a bounded open set in ${ }^{d}$, having two components:

$$
\Omega_{+}=\left\{\left(x_{1}, \ldots, x_{d}\right) \in \Omega_{0}: x_{d}>0\right\},
$$

and

$$
\Omega_{-}=\left\{\left(x_{1}, \ldots, x_{d}\right) \in \Omega_{0}: x_{d}<0\right\} .
$$

We proceed to construct the connected open set $\Omega$ by drilling a central vertical round hole through each diamond $D_{n}$. Let

$$
C_{n}=\left\{(x, y) \in^{d-1} \times:|y|<2 r_{n},\left|x-a_{n}\right|<r_{n}^{1 / r_{n}}\right\} .
$$

Then $C_{n}$ is an open subset of ${ }^{d}$, and using the fact that $r_{n}<1 / 2$ it is easy to check that $C_{n} \subset{ }_{d}(0,1)$ and $C_{n} \cap E=\emptyset$. Let

$$
\Omega=\Omega_{0} \cup \bigcup_{n=1}^{\infty} C_{n} .
$$

Then $\Omega$ is a bounded domaini, and equals the interior of its closure.

Next, we construct the function $f$. We take $f$ to be a $C^{\infty}$ function on $\Omega$ such that $f=1$ on $\Omega_{+}, f=0$ on $\Omega_{-}$, and on each $C_{n}, f\left(x_{1}, \ldots, x_{d}\right)$ depends only on $x_{d}$ and satisfies

$$
\left|\frac{\partial^{k} f}{\partial x_{d}^{k}}\right| \leq \alpha_{k} r_{n}^{-k}
$$


where the constants $\alpha_{k}>0$ depend on $k$, but not on $n$.

\section{The Properties of the Example.}

(3.1) Let $k \in$ and $1 \leq p<+\infty$. Then for each $n$,

$$
\int_{C_{n}}\left|\nabla^{k} f\right|^{p} d x \leq \int_{C_{n}} \alpha_{k}^{p} r_{n}^{-k p} d x
$$

so

$$
\int_{C_{n}}\left|\nabla^{k} f\right|^{p} d x \leq \sum_{n=1}^{\infty} \alpha_{k}^{p} r_{n}^{-k p} \cdot 2 r_{n} \cdot r_{n}^{(d-1) / r_{n}}<+\infty .
$$

Thus $f \in W^{k, p}(\Omega)$.

(3.2) Next, we observe that, given $z, w \in \Omega$, there is a polygonal $\operatorname{arc} \Gamma \subset \Omega$, joining $z$ to $w$, of length at most $5|z-w|$ (- the 5 is not intended to be sharp). It follows that $W^{1, \infty}(\Omega)$ is contained in (and in fact coincides with) $\operatorname{Lip}(1, \Omega)$, and hence each function $g \in W^{1, \infty}(\Omega)$ is uniformly continuous on $\Omega$, and hence extends uniquely to an element of $C(\operatorname{clos}(\Omega))$.

(3.3) The domain $\Omega_{+}$is a Lipschitz domain. Its boundary consists of two sections: a hemisphere, and the graph of a Lip1 finction defined on ${ }_{d-1}(0,1)$, having gradient bounded in norm by 1 . The junction is well-behaved.

(3.4) Let $R$ denote the reflection

$$
R:\left\{\begin{array}{c}
{ }^{d-1} \times \rightarrow^{d-1} \times, \\
(x, y) \mapsto(x,-y) .
\end{array}\right.
$$

Suppose that there exists a sequence $\left\{g_{n}\right\} \subset W^{1, \infty}(\Omega)$ such that $\left\|f-g_{n}\right\|_{W^{1,1}(\Omega)} \rightarrow 0$. Then $\left\|f-g_{n}\right\|_{W^{1,1}\left(\Omega_{+}\right)} \rightarrow 0$, and

$$
\left\|f \circ R-g_{n} \circ R\right\|_{W^{1,1}\left(\Omega_{+}\right)}=\left\|f-g_{n}\right\|_{W^{1,1}\left(\Omega_{-}\right)} \rightarrow 0 .
$$


By the theory of traces of BV functions (cf. [9, (5.10.7), p.259]), there is a continuous linear map

$$
\rho: W^{1,1}\left(\Omega_{+}\right) \rightarrow L^{1}\left(E, \mathcal{H}^{d-1}\right)
$$

such that $\rho(g)=g \mid E$ whenever $g \in C\left(\cos \Omega_{+}\right) \cap W^{1,1}\left(\Omega_{+}\right)$. This fact rests upon the Lipschitzian character of the boundary of $\Omega_{+}$, and the fact that $E \subset$ bdy $\Omega_{+}$.

It follows that $\rho\left(g_{n}\right)=\rho\left(g_{n} \circ R\right)$, since $g_{n}$ extends continuously to $\operatorname{clos}(\Omega)$. Also $\rho(f)=1$ and $\rho(f \circ R)=0$. Thus

$$
\begin{aligned}
0 & <\mathcal{H}^{d-1}(E) \\
& =\int_{E} \rho(f)-\rho(f \circ R) d \mathcal{H}^{d-1} \\
& =\|\rho(f)-\rho(f \circ R)\|_{L^{1}(E)} \\
& =\lim _{n \uparrow \infty}\left\|\rho\left(g_{n}\right)-\rho\left(g_{n} \circ R\right)\right\|_{L^{1}(E)} \\
& =0 .
\end{aligned}
$$

This contradiction shows that $f$ does not belong to the closure of $W^{1, \infty}(\Omega)$ in $W^{1,1}(\Omega)$. It follows that for each $k \in$ and $1 \leq p<+\infty$,

$$
f \in W^{k, p}(\Omega) \sim \operatorname{clos}_{W^{k, p}(\Omega)} W^{k, \infty}(\Omega) .
$$

Remark. To obtain the result for $1<p<+\infty$, one could employ the older Lipschitz boundary result of Stein (cf. [8, p. 192, 4.2]). The BV result quoted above allows us to give a clean treatment of the general case. One could also give a direct proof of the desired property. 


\section{Problem.}

As Vodopyanov points out, it now becomes interesting to characterise those domains $\Omega$ such that $W^{k, \infty}(\Omega)$ is dense in $W^{k, p}(\Omega)$.

\section{Acknowledgment.}

I am grateful to L.E. Fraenkel for bringing the problem to my attention and explaining the background, particularly the earlier work of L. Tartar.

\section{References}

1. R.A. Adams. Sobolev Spaces. Academic Press. New York. 1975.

2. C.J. Amick. Approximation by smooth functions in Sobolev spaces. Bull. Lond. Math. Soc. 11 (1979) 37-40.

3. L. E. Fraenkel. On regularity of the boundary in the theory of Sobolev spaces. Proc. Lond. Math. Soc. (3) 39 (1979) 385-427.

4. P. Jones. Quasiconformal mappings and extendibility of functions in Sobolev spaces. Acta Math. 147 (1981) 71-88.

5. T. Kolsrud. Approximation by smooth functions in Sobolev spaces, a counterexample. Bull. Lond. Math. Soc. 13 (1981) 167-9.

6. J. L. Lewis. Approximation of Sobolev functions and related topics. In C.A. Berenstein (ed.), Complex Analysis I. Proceedings, University of Maryland, 1985-86. Lecture Notes in Mathematics, number 1275. Springer. Berlin, Heidelberg, New York. 1987. pp. 223-34. 
7. - . Approximation of Sobolev functions in Jordan domains. Arkiv for Mat. 25 (1987) $255-64$.

8. E.M. Stein. Singular integrals and Differentiablility Properties of Functions. Princeton University Press. Princeton. 1970.

9. W.P. Ziemer. Weakly Differentiable Functions. Sobolev Spaces and Functions of Bounded Variation. Springer. New York, Berlin, London, Paris, Tokyo, Hong Kong. 1989.

draft 4 , with editor's amendments. 